Revue d'histoire de l'Amérique française

PG REVUE D.HISTOIRE DE L'AMÉRIQUE FRANÇAISE

\title{
Taschereau, Hepburn et les relations Québec-Ontario, 1934-1936
}

\section{René Durocher}

Volume 24, numéro 3, décembre 1970

URI : https://id.erudit.org/iderudit/302987ar

DOI : https://doi.org/10.7202/302987ar

Aller au sommaire du numéro

\section{Éditeur(s)}

Institut d'histoire de l'Amérique française

\section{ISSN}

0035-2357 (imprimé)

1492-1383 (numérique)

Découvrir la revue

\section{Citer cet article}

Durocher, R. (1970). Taschereau, Hepburn et les relations Québec-Ontario, 1934-1936. Revue d'histoire de l'Amérique française, 24(3), 341-355.

https://doi.org/10.7202/302987ar d'utilisation que vous pouvez consulter en ligne.

https://apropos.erudit.org/fr/usagers/politique-dutilisation/ 


\title{
TASCHEREAU, HEPBURN ET LES RELATIONS QUÉBEC-ONTARIO, 1934-1936
}

\author{
RENÉ DUROCher \\ Université de Montréal
}

Il existe entre l'Ontario et le Québec une longue tradition de conflits, de rivalités, mais aussi d'échanges et de coopération. La collaboration autant que le désaccord entre ces deux provinces, alors les plus riches et les plus peuplées du Canada, peut exercer une influence décisive sur l'évolution du pays comme en témoigne l'histoire du siècle dernier.

A l'origine même du régime de 1867 se trouve l'opposition radicale du Canada-ouest et du Canada-est qui s'atténuera avec la mise en place d'un régime fédéral. L'idée de fédération implique l'union des deux Canadas mais aussi, dans certains domaines, leur séparation, comme l'apprendra J. A. Macdonald qui, en voulant établir un régime très centralisateur, se heurtera à l'opinion publique ontarienne. Le spectre d'une nouvelle "French Domination" autant que la crainte de devenir "la vache à lait" du Dominion feront de l'Ontario le défenseur énergique des "Provincial Rights".

En Ontario, l'absence d'un nationalisme purement canadian rendait possible l'apparition d'un vigoureux provincialisme, tandis qu'au Québec ce sera le réveil du nationalisme canadienfrançais qui permettra à Honoré Mercier de promouvoir avec succès la cause de l'autonomie provinciale. Même si le Québec et l'Ontario s'opposent vigoureusement à propos de l'affaire Riel, leurs premiers ministres, Honoré Mercier et Oliver Mowat, s'entendent fort bien lors de la conférence interprovinciale de 1887 à Québec. ${ }^{1}$ Cette rencontre au sommet marque le début d'une longue tradition qui, si l'on en croit certains journalistes, se perpétue jusqu'à nos jours... Mais avant de nous embourber dans des spéculations douteuses, revenons en arrière et contentons-nous d'analyser une séquence des rapports Québec-Ontario qui débute avec l'arrivée de Mitchell Hepburn au pouvoir en 1934 et qui se termine avec la démission d'Alexandre Taschereau en 1936.

$1 \mathrm{~J}$. C. Morison, Oliver Mowat and the Development of Provincial Rights in Ontario: A Study in Dominion-Provincial Relations 1867-1896 (Toronto, 1961), 234-285.

[ 341 ]

RHAF, vol. 24, no 3 (décembre 1970) 


\section{Mitchell Hepburn}

En 1926, le parti libéral fédéral laisse le jeune Hepburn briguer les suffrages auprès des électeurs du comté d'Elgin-ouest qui depuis vingt-cinq ans élisait un conservateur pour les représenter à Ottawa. A la surprise générale, le "farmer" Hepburn, âgé de trente ans seulement, remporte la victoire et devient le plus jeune député à la Chambre des Communes. "Mitch", comme on l'appelle à St-Thomas, s'intéresse aux problèmes des agriculteurs qu'il connaît bien car, en plus de l'expérience acquise sur la ferme paternelle, il a occupé pendant trois ans le poste de secrétaire des United Farmers of Ontario d'Elgin-est. Il prête aussi beaucoup d'attention aux questions économiques et financières qu'il a étudiées en autodidacte après son expulsion du collège de St-Thomas.

En Chambre, il se fait remarquer par ses attaques répétées contre la compagnie d'assurances Sun Life, ce qui agace certains de ses collègues libéraux. Malgré cet écart et queloques sautes d'humeur à propos du patronage dans son comté, il reste un partisan loyal. Alors que plusieurs libéraux réprouvent le fameux "five-cents speech" de Mackenzie King, Hepburn défend la position de son chef et il renchérit en disant qu'à la place du premier ministre il aurait rabroué encore plus durement le gouvernement tory d'Ontario qui demandait au gouvernement fédéral de prendre des mesures adéquates pour faire face à la crise. A l'élection de 1930 , alors même que King perd son siège en Ontario, Hepburn est l'un des rares survivants de son parti dans cette province et prouve ainsi son habileté de politicien.

A cette époque, les libéraux provinciaux d'Ontario occupent les banquettes de l'opposition depuis 1905 et, après sept défaites successives sous la direction de huit leaders différents, ils sont complètement démoralisés. Dans un geste désespéré, ils demandent à Hepburn de prendre la direction du parti. De 1930 à 1934 , tout en conservant son poste à Ottawa, Hepburn réorganise le parti et prononce de nombreux discours à travers l'Ontario. Le 19 juin 1934, contre toute attente, Hepburn remporte une victoire décisive en faisant élire soixante-cinq libéraux dans les quatre-vingt-dix circonscriptions de la province et il devient, à trente-sept ans, le plus jeune premier ministre de l'Ontario. ${ }^{2}$

Le Devoir félicite le vainqueur et se plaît à souligner que sur les quatre-vingt dix élus on dénombre sept députés franco-

2 Pour plus de détails sur la carrière de Hepburn, cf. Neil McKenty, Mitch Hepburn (Toronto, 1967), 307 pages. 
ontariens (tous libéraux) tandis que précédemment, avant le remaniement de la carte électorale, on n'en comptait que cinq sur cent douze. De plus, M. Hepburn a promis de nommer un ministre canadien-francais avec portefeuille contrairement au gouvernement conservateur de Henry qui n'avait pas confié de responsabilité définie au seul représentant franco-ontarien qui siégeait dans son cabinet. ${ }^{3}$

Le premier ministre Taschereau, songeant sans doute aux élections qui devront avoir lieu l'année suivante, émet l'opinion que la victoire des libéraux en Ontario et en Saskatchewan provient $d u$ fait que les deux gouvernements conservateurs de ces provinces étaient associés au gouvernement Bennett discrédité auprès de l'opinion publique canadienne. Maurice Duplessis, chef du parti conservateur, se sent visé par cette interprétation partisane et réplique d'une manière cinglante:

A l'avenir les élections provinciales vont se faire sur le provincial. Il est fini le beau temps où $M$. Taschereau gagnait ses élections en les faisant sur le dos du fédéral et je le préviens dès aujourd'hui ... Les électeurs d'Ontario ont voulu se choisir un jeune chef et un jeune gouvernement. Toutes les critiques que M. Hepburn a faites du gouvernement Henry pourraient s'appliquer au gouvernement Taschereau. M. Hepburn n'a rien de commun avec M. Taschereau. ${ }^{4}$

\section{Taschereau-Hepburn}

Le premier ministre du Québec qui entretenait d'excellentes relations avec les dirigeants conservateurs de l'Ontario depuis 1920 espère continuer cette politique avec le nouveau leader libéral. L'allégeance politique commune des deux hommes devrait faciliter les choses d'autant plus que les deux provinces centrales sont confrontées à des problèmes analogues. Le 14 septembre 1934, à la veille d'une conférence fédérale-provinciale, Taschereau essaie d'organiser une réunion des dirigeants de la NouvelleEcosse, de l'Ontario et du Québec. Il écrit à Hepburn:

What do you think of yourself, Hon. Mr. Macdonald, P.M. of N.S. and myself meeting at some later date to discuss the situation and arrive at a conclusion which would permit us to offer a united front? I believe that Ontario, the Maritimes and Quebec have some commun interests to protect and should agree on a common policy. ${ }^{5}$

${ }^{3}$ Le Devoir, 20 juin 1934.

4 Ibid., 21 juin 1934.

5 L.-A. Taschereau à M. F. Hepburn, 14 septembre 1934. Hepburn Papers, Private, 1934, Provincial Archives of Ontario (Toronto). 
Hepburn écrit à Macdonald pour l'informer qu'il accepte le projet de "our mutual friend Premier Taschereau". 6 Nous n'avons trouvé aucun indice nous permettant d'affirmer que la rencontre eut lieu ou non mais il importe de remarquer la cordialité des relations Taschereau-Hepburn et, surtout, l'idée de former un front commun contre Ottawa.

Avec ou sans consultations, les politiques des deux premiers ministres se rejoignent sur plusieurs points à la fin de 1934 et au début de 1935. Ainsi Taschereau, qui depuis plusieurs années dénonce le projet de canalisation du Saint-Laurent, apprend avec plaisir que son collègue ontarien ne partage pas les vues du gouvernement conservateur Henry qui avait donné son accord de principe au projet canado-américain. ${ }^{7}$ En décembre, alors que Hepburn dénonce à nouveau le coûteux et inutile projet de canalisation, Taschereau déclare aux journalistes: "Je suis heureux de voir que M. Hepburn se joint à nous pour s'opposer à la canalisation du Saint-Laurent. Cela nous est d'un apport considćrable. J'espère que le projet ne sera pas enterré, mais noyé." 8

Taschereau a sans aucun doute apprécié encore plus l'appui de Hepburn dans sa lutte contre l'"American Newspaper Publishers". Depuis le début de la crise, l'industrie papetière connaît des difficultés et la surproduction a fait tomber les prix à un niveau inquiétant. Taschereau exerce des pressions sur les compagnies pour que d'un commun accord elles fixent le prix du papierjournal à $\$ 45.00$ la tonne. La St. Lawrence Paper refuse de suivre les autres compagnies et compromet ainsi le projet. Le président de l'“American Newspaper Publishers" tente d'intimider Taschereau en déclarant qu'il demandera aux journaux américains d'acheter leur papier ailleurs qu'au Canada. ${ }^{9}$ Hepburn intervient publiquement pour aider son collègue québecois.

The Government of Ontario will take no advantage of Quebec's difficulty, nor of the resentment which the publishers have expressed. Our government is fully in accord with Premier Taschereau's attitude and if necessity arise we shall do our full duty in a similar way. ${ }^{10}$

G M. F. Hepburn à A. Macdonald, 15 octobre 1934. Hepburn Papers, Private, 1934.

7 Le Devoir, 24 octobre 1934.

8 Ibid., 4 décembre 1934.

9 Robert Rumilly, Histoire de la province de Québec (Montréal, 1963), 34: 96. Aussi Le Canada, 13, 18 et 25 octobre 1934.

10 Newspapers Industry. Hepburn Papers, Private, 1934. 
Le mois suivant, Hepburn proteste contre le projet du fédéral de lever un impôt sur les mines d'or et Taschereau, qui depuis plusieurs mois dénonçait cette tentative du gouvernement Bennett, appuie fermement le premier ministre ontarien car, dit-il: "Il est injuste qu'Ottawa impose la production de l'or dans les provinces. Autant dire qu'il a le droit d'imposer l'exploitation de nos forêts, de nos lacs, et de toutes nos richesses naturelles." ${ }^{11}$ La collaboration entre les deux provinces se fait encore plus précise dans le domaine du travail. Les deux ministres responsables de cette question se rencontrent et $M$. Roebuck, le ministre ontarien, songe à adopter une loi semblable à "l'Extension juridique des conventions collectives". Effectivement en 1935, l'Ontario votera l' Industrial Standard Act" qui permettra d'établir dans quelques industries une quasi-parité des salaires dans les deux provinces.

Dans cette période de crise économique, le problème des finances provinciales cause beaucoup de soucis aux deux premiers ministres qui se consultent sur certaines questions. Ainsi, le Québec fait part à l'Ontario de son désir de hausser d'un ou deux cents la taxe sur l'essence. Devant le refus de Hepburn d'augmenter cette taxe, Taschereau consent à chercher d'autres sources de revenu. ${ }^{12}$ Ce problème réglé, Taschereau écrit de nouveau à Hepburn pour lui rappeler qu'au temps de son prédécesseur il y avait entente entre les deux provinces pour refuser aux municipalités le droit d'accorder aux compagnies certaines exemptions d'impôts. Le premier ministre affirme qu'il a reçu plusieurs plaintes à l'effet que des municipalités du Québec ont perdu certaines industries par suite d'une concurrence déloyale des municipalités ontariennes. Hepburn répond qu'il est parfaitement d'accord pour arrêter cette concurrence ruineuse entre les deux provinces. Il ajoute que les industriels faisaient du chantage puisqu'ils disaient aux municipalités de sa province qu'à défaut d'obtenir les exemptions demandées ils iraient s'établir au Québec qui les leur accorderait. ${ }^{13}$ Les deux premiers ministres essaient, tant bien que mal, de trouver des moyens de régler le problème crucial du chômage chronique qui grève les budgets provinciaux. Sans s'être entendus au préalable, ils dénoncent l'ineptie du gouvernement Bennett et tous deux, à

${ }^{11}$ Le Canada, 7 novembre 1934.

12 Taschereau-Hepburn, correspondance du 6 décembre 1934 au 16 janvier 1935. Hepburn Papers, Public, 1935.

13 Taschereau-Hepburn, correspondance du 18 janvier au 8 février 1935. Hepburn Papers, Public, 1935. 
un mois d'intervalle, en viennent à demander au fédéral de se retirer complètement du champ de l'impôt direct. ${ }^{14}$

Malgré ces déclarations qui se rejoignent et ces consultations dont nous venons de parler, les relations Québec-Ontario se détériorent au début de 1935, comme en témoignent les opinions différentes de MM. Hepburn et Taschereau à propos de l'industrie du papier-journal. Les pressions de Taschereau pour obliger les producteurs de papier à fixer un prix convenable pour leur produit s'avérant inefficaces, il avait eu un entretien avec Peter Heenan, le ministre ontarien des terres et forêts. Après cette première rencontre, Taschereau essaie à deux reprises, mais sans succès, de rencontrer Heenan pour mettre au point une stratégie commune aux deux provinces. Ne pouvant attendre indéfiniment, il prépare un projet de loi "pour assurer la protection des ressources forestières de la province", 15 et en fait parvenir une copie à Heenan pour connaître son avis. Celui-ci ne daigne même pas répondre et Taschereau s'adresse directement à Hephurn nour se plaindre de la mauvaise volonté de son ministre et lui décrire la situation dans l'industrie du papier.

[It] is almost desperate. We cannot depend on the industry itself to get over its trouble and I never met a group of men so disloyal to each other... unless Ontario and Quebec will join in a common action, I believe that matters will become from bad to worse and a most ruinous competition will arise between both provinces. I see it coming. ${ }^{16}$

Hepburn répond qu'il est d'accord sur le but à atteindre mais non sur les moyens coercitifs que veut employer le premier ministre du Québec pour contraindre les compagnies récalcitrantes à se mettre au pas. "I am entirely in sympathy with your desire to obviate the self destruction activities of the newsprint producers in the province, but I think more can be gained through conciliation in the manner suggested by the Hon. Peter Heenan." 17

Le différend entre les deux chefs libéraux s'explique, en partie, par les illusions de Hepburn qui pense pouvoir convaincre les compagnies de papier de maintenir un prix raison-

${ }_{14}$ Le Canada, 4 mars 1935 et Debates of the Legislative Assembly of Ontario (Newspaper Hansard on Microfilm), 3 avril 1935.

15 Statuts de la province de Québec, chapitre 22 , 4e session, $18 \mathrm{e}$ législature, 25-26 Geo V, 1935.

16 L.-A. Taschereau à M. F. Hepburn, 12 mars 1935. Hepburn Papers, Private, 1935.

${ }_{17}$ M. F. Hepburn à L.-A. Taschereau, 18 mars 1935. Hepburn Papers, Private, 1935. 
nable, d'engager le plus grand nombre possible de bûcherons et de les payer convenablement moyennant une réduction des droits de coupe par le gouvernement ontarien. Une autre raison, au moins aussi importante que la première et dont il ne parle pas dans sa lettre, c'est sa conviction que le Québec profite de l'Ontario, grâce à quatre compagnies d'électricité québecoises qui font affaire avec l'Hydro-Ontario.

Three Ontario paper mills are closed down, [dit M. Hepburn] while Quebec mills continue to operate and the reason is that the power companies in the Eastern Province largely through the revenues derived from the sale of electrical energy to this Province, are able to drive Ontario mills to the wall. 18

Dans les années '20, l'Ontario en pleine croissance économique craignait de manquer d'électricité et le moyen le plus économique de pallier à cette éventuelle pénurie consistait à en acheter des compagnies privées du Québec. La crise de '29 bouleverse tous les calculs des experts et l'Ontario dispose alors d'un surplus d'électricité considérable. Les contrats qui paraissaient raisonnables au temps de la prospérité deviennent très onéreux dans la conjoncture des années ' 30 . Hepburn avait promis de réaliser des économies et l'un des moyens d'atteindre cette fin consistait à renégocier de nouveaux contrats avec les compagnies d'électricité. Devant le refus de celles-ci de rogner leur marge de profit, Hepburn présente une loi qui déclare que les contrats signés par l'Hydro-Ontario étaient et avaient toujours été "illegal, void and unenforcable". ${ }^{19} \mathrm{Il}$ annonce que la loi entrera en vigueur lorsque le lieutenant-gouverneur la sanctionnera. En d'autres mots, il laisse planer sur la tête des quatre compagnies une épée de Damoclès pour les obliger à négocier de nouveaux contrats.

Pendant qu'on discute du projet de loi à l'Assemblée législative, des négociations intenses se déroulent dans le bureau du premier ministre. Les quatre compagnies impliquées délèguent un avocat auprès du premier ministre ontarien de même que le gouvernement Taschereau envoie M. Gordon Scott pour défendre les intérêts de la province de Québec.

Hepburn racontera plus tard ${ }^{20}$ que, lors de ces négociations, le représentant de Taschereau aurait menacé le gouvernement

18 Debates of the Legislative Assembly of Ontario, 8 mars 1935.

19 Statutes of Ontario, Power Commission Act, Chapter 53, First Session of the Nineteenth Legislature of Ontario, 25 Geo V, 1935.

20 Globe and Mail, 18 décembre 1936. 
ontarien de priver certaines régions de sa province d'électricité. Cela aurait été extrêmement grave dans certaines régions ontariennes où l'Hydro ne pouvait produire d'énergie à 60 cycles. Il est impossible de savoir si cette menace a été réellement proférée, mais on est enclin à accepter la parole de Hepburn puisqu'il a fait installer en toute hâte un transformateur de fréquence à Chats Falls au coût d'un million de dollars! De plus, la réponse de l'ex-premier ministre du Québec à cette accusation peut laisser planer quelques soupçons dans notre esprit... M. Taschereau dit qu'il doute que la province ait le droit d'empêcher le commerce interprovincial. Cet argument d'avocat n'est guère probant, car il suffirait de laisser les compagnies d'électricité en cause prendre l'initiative de ce boycottage pour arriver au même résultat. Quant au rôle de Gordon Scott, voici ce qu'il en dit:

J'ignore ce qui s'est passé à Toronto n'ayant pas assisté à la conférence, mais l'hon. $M$. Scott m'autorise à dire qu'il n'avait pas reçu instruction de faire une telle menace et qu'il ne l'a faite en aucun temps.

Il est possible qu'il ait dit que l'opinion publique était très émue à Québec, que le bon voisinage était en danger, que la riposte était possible, mais encore une fois, j'ignore si M. Scott a tenu ce langage. ${ }^{21}$

Quoi qu'il en soit, au début d'avril, l'Assemblée législative vote la loi résiliant les contrats d'électricité. Taschereau dénonce cette mesure et tente à nouveau, mais sans succès, de négocier une entente avec Hepburn. Il annonce tout de même qu'après la session il rencontrera son collègue ontarien pour discuter du problème de la double taxation sur les droits de succession, de la loi des compagnies, de la question du papier et bien entendu des contrats d'électricité. Du 23 mai au 28 juin les deux premiers ministres tentent en vain de fixer une date pour se rencontrer. Finalement, Taschereau écrit à son collègue:

... it has been suggested to me that we might postpone indefinitely the meeting on account of the approaching dissolution of Parliament. Our Conference may cause some dissatisfaction, either in Ontario or in Quebec, as it is perhaps difficult to settle some of our problems without some friction. ${ }^{22}$

Hepburn accepte volontiers cette suggestion et il ajoute: "I quite concur that in view of the approaching federal election,

${ }^{21}$ La Presse, 19 décembre 1936.

${ }^{22}$ L.-A. Taschereau à M. F. Hepburn, 3 juillet 1935, Hepburn Papers, Private, 1935. 
it would be well for us to have as little publicity as possible with respect to some of the problems which we have to settle." ${ }^{23}$ C'est ce qui explique qu'après la tension très forte de marsavril les relations semblent s'améliorer entre les deux chefs libéraux qui mettront leurs énergies au service de Mackenzie King qui tente d'enlever le pouvoir au gouvernement Bennett. Le 30 septembre, à Québec, en présence de 5,000 personnes, Hepburn vient prononcer un discours en faveur de King et du parti libéral.

Le correspondant du Devoir nous rapporte "[qu'] on avait hâte d'entendre ce premier ministre dont les dépêches ont parlé si souvent depuis un an ... A son entrée dans la salle, $M$. Hepburn a été l'objet d'une longue ovation." ${ }^{24}$ Le premier ministre du Québec, qui, au dire même de Hepburn, "dirige avec tant d'habileté les destinées de la province", 25 se déclare très heureux à la suite de cette magnifique soirée. On a l'impression que dans l'euphorie de la campagne électorale, Taschereau a presque oublié ses griefs contre le leader ontarien qu'il se propose de rencontrer après les élections fédérales afin de régler, à l'amiable, les problèmes entre les deux provinces-sœurs. Effectivement, peu après la victoire des libéraux à Ottawa, Honoré Mercier, le ministre des terres et forêts du Québec, rencontre Hepburn à Toronto et celui-ci semble prêt à poursuivre les négociations avec les compagnies d'électricité. Mais au début de décembre, rien ne va plus, les négociations sont rompues. Hepburn demande au lieutenant-gouverneur de sanctionner la loi annulant les contrats intervenus entre l'Hydro-Ontario et les quatre compagnies québecoises qui avait été votée par l'Assemblée législative en avril 1935. Une semaine après cet incident, Taschereau rencontre son collègue ontarien à Ottawa à l'occasion de la conférence fédérale-provinciale de décembre 1935. Les deux premiers ministres profitent de l'occasion pour tenir une réunion privée et discuter à nouveau de la question de l'électricité. Il semble que les deux hommes ont échangé des propos peu amènes sur ce sujet, comme nous le révèlera plus tard le premier ministre ontarien.

The matter came to a climax when, at the Dominion-Provincial Conference at Ottawa, $\mathrm{Mr}$ Taschereau, in the presence of the Attorney General, the Hon. Peter Heenan, Chester Walters and myself, again threatened that unless we took all

${ }^{23}$ M. F. Hepburn à L.-A. Taschereau, 5 juillet 1935. Hepburn Papers, Private, 1935.

24 Le Devoir, 1er octobre 1935.

${ }^{25}$ Le Canada, 1er octobre 1935. 
the power he would shutt off all export to Ontario, and intimated further that he understood plans were underway to commit sabotage by pulling the plugs on the sluice gates on the Quebec side of the Chats Falls development, thus rendering Ontario development at that place useless. ${ }^{26}$

Taschereau, qui depuis quelques mois avait quitté la scène politique, remet un communiqué aux journalistes pour expliquer que la déclaration de Hepburn repose sur un malentendu et que jamais il n'a proféré de menaces contre l'Ontario. Voici, selon l'ex-premier ministre du Québec, quelques-uns des propos qu'il aurait échangés "très amicalement" avec son collègue ontarien à Ottawa en décembre 1935.

Je répétai à $M$. Hepburn que sa répudiation des contrats créait une très mauvaise impression dans Québec, que les actionnaires y perdraient beaucoup et que l'opinion publique pourrait tenter de forcer le gouvernement à prendre quelque action draconienne. J'ajouterai que, en aucun temps, nous n'avons pris ni eu l'intention d'adopter une telle ligne de conduite.

[Quant à la menace de sabotage à Chats Falls, il aurait dit:] Je comprends que la compagnie de Québec [Ottawa Valley Power] a fait savoir que si ses contrats étaient annulés elle serait sans marchés pour son pouvoir [de Chats Falls] et ouvrirait dès lors, les vannes de son côté de la rivière, ce qu'elle avait le droit de faire. On m'a fait connaître cette intention et j'en ai fait part à $M$. Hepburn, mais encore une fois, notre gouvernement n'était et ne pouvait être partie à cette manœuvre. ${ }^{27}$

Nous ne nous permettrons pas de dire comme M. Hepburn que Taschereau ment ${ }^{28}$, mais sa déclaration ambiguë ne nous paraît pas très convaincante.

Le conflit entre Taschereau et Hepburn sur la résiliation des contrats d'électricité les empêche d'unir leurs efforts contre le gouvernement fédéral lors de cette conférence fédérale-provinciale. A part la question de la conversion obligatoire des dettes publiques, qui constitue pour Hepburn une panacée aux problèmes financiers du pays et que Taschereau rejette pour des raisons d'orthodoxie financière, ${ }^{29}$ les deux provinces centrales

26 Globe and Mail, 18 décembre 1936.

27 La Presse, 19 décembre 1936.

28 Globe and Mail, 22 décembre 1936.

29 Draft Minutes of the Sub-Conference on Financial Questions. Hepburn Papers, Private, 1935. 
sont généralement d'accord sur chacun des problèmes abordés lors de ces assises.

Cependant, et il importe de le souligner, la réaction globale des deux premiers ministres provinciaux diffère profondément, face aux tentatives du gouvernement King pour trouver une solution aux problèmes qui assaillent le pays. Ainsi, Hepburn est tellement insatisfait de l'attitude des fédéraux qu'il quitte la conférence avant la fin des rencontres, tandis que Taschereau se montre très conciliant et, somme toute, très satisfait des résultats obtenus. ${ }^{30}$ Il faudra attendre l'arrivée au pouvoir de Duplessis pour que prenne forme l'alliance du Québec et de l'Ontario contre le gouvernement central.

Malgré l'affrontement entre les deux premiers ministres provinciaux à l'occasion de la conférence fédérale-provinciale, l'interdépendance des deux provinces centrales est telle, surtout en période de crise économique, qu'en février 1936, M. Peter Heenan, le ministre ontarien des terres et forêts, vient rencontrer MM. Taschereau et Mercier pour tenter encore une fois d'établir une politique commune aux deux provinces touchant l'industrie du papier. Taschereau préconisait une intervention directe du gouvernement dans ce domaine, mais du côté ontarien on voulait encore essayer la persuasion et les pressions indirectes. Taschereau souhaitait mettre fin à la concurrence entre les compagnies et entre les deux provinces en fixant un prix unique pour le papier-journal. Heenan craint d'aller aussi loin. Afin de réduire la concurrence, on convient tout de même "de supprimer les différences qui existent actuellement, d'une province à l'autre, entre les salaires et les heures de travail". 31

Taschereau, généralement très courtois et surtout très partisan, avait toujours évité d'attaquer publiquement le gouvernement libéral d'Ontario. Mais après le demi-échec de la rencontre avec Heenan et surtout après son échec pour empêcher la "répudiation" des contrats d'électricité, il éclate et dénonce, non sans quelque démagogie, la politique ontarienne.

... l'Ontario vient de répudier des contrats. Libre à elle. Mais la province de Québec ne l'a pas fait, et j'espère qu'elle ne le fera pas. De plus l'Ontario vient de faire une loi pour imposer une taxe sur le revenu de ses citoyens; il y a donc, dans la province voisine, un impôt sur le revenu, prélevé et par le fédéral et par le provincial. Si nous pouvons montrer

${ }^{30}$ Conférence du Dominion et des provinces. Compte rendu des délibérations, Ottawa, 9-13 décembre 1935 (Ottawa, 1936), 47-50.

31 Le Canada, 26 février 1936. 
à ceux qui possèdent du capital que la province de Québec leur donne des garanties de tout repos, qu'ils trouveront chez elle un travail sain et des bras vigoureux, ne croit-on pas qu'il en résultera beaucoup de bien pour la province... Si nous montrons aux industriels et aux commerçants que dans la province de Québec, on ne répudie pas les contrats, on n'écrase pas les gens sous le fardeau des impôts, ne croit-on pas que le capital viendra chez-nous établir des industries. ${ }^{32}$

Cette attaque virulente de Taschereau contre Hepburn nous paraît tout à fait exceptionnelle dans l'histoire des relations Québec-Ontario depuis 1867. En général, les dirigeants des deux provinces centrales entretenaient de bonnes relations ou tout au moins ne s'attaquaient pas publiquement lorsque leurs politiques divergeaient profondément ou même lorsque l'opinion publique des deux provinces se déchaînait sur des problèmes ethniques comme l'affaire Riel, le règlement 17 , la crise de la conscription, etc.

Cette dernière intervention de Taschereau, pas plus que les précédentes, ne modifiera la politique de Hepburn. Pis encore, elle ne réduira même pas l'étonnante popularité du premier ministre ontarien au Québec.

\section{La popularité de Hepburn au Québec}

En 1934 les Franco-Ontariens avaient appuyé massivement Hepburn qui pendant la campagne électorale avait promis de prendre en considération la demande des catholiques ontariens qui réclamaient une juste répartition des impôts scolaires entre les écoles publiques et séparées. Les conservateurs ne voulaient pas se compromettre et proposaient de référer toute cette question aux tribunaux. L'affaire du règlement 17 ne permettait plus aux catholiques d'espérer quoi que ce soit de ce côté et les sept députés franco-ontariens élus en 1934 représentaient tous le parti libéral.

Une fois au pouvoir, Hepburn reçoit avec cordialité les représentants de la "Catholic Tax Payers Association" et, selon le premier ministre, les catholiques ne demandent au fond qu'un traitement équitable comme celui qu'on accorde aux protestants dans le Québec. Les adversaires de la réforme organisent une imposante délégation comprenant, entre autres, deux cents orangistes, pour protester contre toute modification du partage de l'impôt scolaire. Hepburn recoit assez froidement les orangistes et les avertit qu'il ne se laissera pas intimider par eux.

\footnotetext{
${ }_{82}$ Le Devoir, 27 mars 1936.
} 
Les journaux canadiens-français suivent de près cette question et vantent sans réserve le courage de Hepburn. Omer Héroux reflète bien le sentiment de ses compatriotes lorsqu'il écrit: "Il y a pour l'homme qui aura le courage de régler cette question [scolaire], une bel'e place à prendre dans la galerie des bienfaiteurs du pays, des bons ouvriers de la paix nationale." ${ }^{33}$

En 1936, après des débats orageux à l'Assemblée législative ontarienne, le premier ministre fait voter une loi assurant aux catholiques une partie des impôts payés par les compagnies privées. Cette loi n'améliorait que très partiellement la situation financière des écoles séparées, puisque les compagnies de la couronne continueront de verser leurs impôts aux seules écoles publiques et surtout cette loi était soumise à une procédure qui en rendait l'application très difficile. La controverse fait rage à un point tel que, l'année suivante, Hepburn retire la loi sur l'impôt scolaire pour éviter, dit-il, une guerre de religion en Ontario. Les Canadiens français sont fort déçus, mais d'une manière générale, ils ne blâment pas Hepburn qui a fait preuve de bonne volonté et de courage dans cette controverse.

Le Québec, dominé par le régime Taschereau, fatigué, prudent et orthodoxe, souhaiterait trouver un "radical" comme Hepburn, pour le sortir du marasme économique. Mitchell Hepburn est jeune, dynamique, sincère et d'une brutale franchise qui suscite l'enthousiasme des Québecois. Il ne craint pas de s'attaquer aux "trusts" et aux puissances financières. Sa "répudiation" des contrats d'électricité lui a valu une immense popularité dans les milieux réformistes du Québec qui luttaient avec acharnement contre le trust québecois de l'électricité. Oscar Drouin de l'A.L.N. traduit le sentiment de plusieurs lorsqu'il s'écrie dans un discours à la radio: "Nous avons besoin d'un Hepburn dans la province de Québec pour casser les reins de la dictature économique." 34

Chaque semaine, ou presque, la presse rapporte quelques gestes spectaculaires du premier ministre ontarien. Ainsi, dès son arrivée au pouvoir, il opère un grand nettoyage à Queen's Park en congédiant plusieurs fonctionnaires qui, dit-il, continueront à ne rien faire mais sans être payés pour leur oisiveté comme au temps des conservateurs. Il abolit plusieurs commissions, notamment celle de la fonction publique qui, selon lui, est inutile, puisque depuis toujours les ministres et sous-ministres choisissaient directement les fonctionnaires. Il déclenche une

33 Ibid., 29 juin 1935.

34 Ibid., 26 juin 1935. 
série d'enquêtes sur l'administration précédente et pendant quelques mois les conservateurs se trouvent dans une situation difficile.

Pendant sa campagne électorale Hepburn a promis de réaliser des économies et, une fois devenu premier ministre, il entend tenir sa promesse ! Il réduit le salaire des ministres; devant cinq mille personnes il vend à l'encan les quarante-sept limousines du gouvernement pour une somme d'environ trentequatre mille dollars; il ferme la maison de l'Ontario à Londres; il demande au lieutenant-gouverneur de démissionner pour raison d'économie! Ces gestes ne signifient pas grand-chose au point de vue économique mais ils frappent l'imagination populaire.

S'il est vrai que l'Ontario se distingue par son anglophilie, voire son impéralisme, Mitchell Hepburn est un Ontarien singulier puisque, en plus des gestes déjà mentionnés à propos du poste de lieutenant-gouverneur et de la maison de l'Ontario à Londres, il se déclare isolationniste. Plusieurs Québecois se réjouissent d'entendre Hepburn déciarer "qu'il ne croit pas que le Canada se laisse entraîner à participer à une nouvelle guerre européenne".

Le Québec obsédé par la "menace communiste" ne manque pas de noter avec satisfaction l'opposition de Hepburn à cette doctrine et sa sympathie pour le catholicisme. "Les adversaires de la réforme scolaire accusent le catholicisme de tous les maux. Laissez-moi vous dire que le plus grand mal à craindre est le communisme et que l'Eglise catholique est le rempart contre le communisme." 36

Les nationalistes québecois qui, depuis toujours, dénoncent l'esprit de parti comme un fléau, ne sont pas choqués de voir Hepburn différer d'opinion avec son chef Mackenzie King lorsqu'il y va de l'intérêt de l'Ontario. Aux yeux de certains, Hepburn apparaît même comme un défenseur plus vigoureux de l'autonomie provinciale que le premier ministre du Québec.

Le premier ministre d'Ontario jouit d'une telle popularité au Québec qu'à la veille de l'élection provinciale d'août 1936 , Ernest Lapointe demande à ses compatriotes de voter pour Adélard Godbout, qui vient de succéder à Taschereau et qu'il compare à cet autre agriculteur et chef libéral: Mitchell Hepburn ! ${ }^{37}$ Malgré les efforts des libéraux, les Québecois vote-

35 Ibid., 15 septembre 1936.

36 Ibid., 6 mai 1936.

37 Ibid., 15 août 1936. 
ront pour Maurice Duplessis qui, à plusieurs points de vue, ressemble bien plus à Hepburn que Godbout ou son prédécesseur.

Le nouveau premier ministre du Québec, Maurice Duplessis, aura des relations très cordiales et très suivies avec son homologue ontarien. Les libéraux, qui s'inquiétaient des mauvaises relations Taschereau-Hepburn, s'inquiéteront bien davantage de ce qu'on a appelé "l'alliance Duplessis-Hepburn" qui de 1936 à 1939 tentera de s'opposer à l'évolution du fédéralisme canadien dans un sens centralisateur. 